\title{
Outcomes Impacting Quality of Life in Advanced Parkinson's Disease Patients Treated with Levodopa-Carbidopa Intestinal Gel
}

\author{
Norbert Kovács ${ }^{\mathrm{a}, *}$, Lars Bergmann ${ }^{\mathrm{b}}$, Marieta Anca-Herschkovitsch ${ }^{\mathrm{c}}$, Esther Cubo ${ }^{\mathrm{d}}$, \\ Thomas L. Davis ${ }^{\mathrm{e}}$, Robert Iansek ${ }^{\mathrm{f}}$, Mustafa S. Siddiqui ${ }^{\mathrm{g}}$, Mihaela Simu ${ }^{\mathrm{h}}$, David G. Standaert ${ }^{\mathrm{i}}$, \\ K. Ray Chaudhuri ${ }^{\mathrm{j}, \mathrm{k}}$, Paul Bourgeois ${ }^{1}$, Tianming Gao ${ }^{\mathrm{b}}$, Pavnit Kukreja ${ }^{\mathrm{b}}$, \\ Francesco E. Pontieri $^{\mathrm{m}, \mathrm{n}}$ and Jason Aldred $^{\mathrm{O}}$ \\ ${ }^{a}$ University of Pécs, Medical School, Pécs, Hungary \\ ${ }^{\mathrm{b}}$ AbbVie Inc., North Chicago, IL, USA \\ ${ }^{\mathrm{c}}$ Edith Wolfson Medical Center, Holon, Israel \\ ${ }^{\mathrm{d}}$ Neurology Department, Hospital Universitario Burgos, Burgos, Spain \\ ${ }^{\mathrm{e}}$ Vanderbilt University Medical Center, Nashville, TN, USA \\ ${ }^{\mathrm{f}}$ Kingston Centre, Monash Health, Melbourne, Australia \\ ${ }^{\mathrm{g}}$ Wake Forest School of Medicine, Winston Salem, NC, USA \\ ${ }^{\mathrm{h}}$ Victor Babes University of Medicine and Pharmacy, Timisoara, Romania \\ ${ }^{\mathrm{i}}$ University of Alabama at Birmingham, Birmingham, AL, USA \\ jParkinson's Foundation International Centre of Excellence, King's College Hospital, London, UK \\ ${ }^{\mathrm{k}}$ King's College Institute of Psychiatry, Psychology \& Neuroscience, London, UK \\ ${ }^{1}$ Department of Neurology AZ Groeninge, Kortrijk, Belgium \\ ${ }^{\mathrm{m}}$ Sapienza University of Rome, Rome, Italy \\ ${ }^{\mathrm{n}}$ Fondazione Santa Lucia, IRCSS, Rome, Italy \\ ${ }^{\circ}$ Selkirk Neurology, Spokane, Washington, USA
}

Accepted 17 December 2021

Pre-press 30 December 2021

\begin{abstract}
.
Background: It is believed that motor symptoms, including dyskinesia, and non-motor symptoms impact health-related quality of life (HRQoL) in patients with Parkinson's disease (PD), and that improvements in these metrics are correlated. Objective: Investigate the relationship between HRQoL and measures of PD severity and treatment efficacy, including motor and non-motor symptoms.

Methods: This was a planned investigation of an international, prospective, single-arm, post-marketing observational study of the long-term effectiveness of levodopa-carbidopa intestinal gel (LCIG) in patients with advanced PD. Pearson correlation coefficients (PCC) were calculated for baseline and change from baseline at 12 months between HRQL and motor and non-motor symptoms.
\end{abstract}

*Correspondence to: Norbert Kovács, MD, PhD, Department of Neurology, University of Pécs, 7623 Pécs, Rét u. 2., Pécs,
7623 Hungary. Tel.: +36 72535 910; E-mail: norbert.kovacsdr@ gmail.com; ORCID: 0000-0002-7332-9240 
Results: A total of 195 patients were included. At baseline, HRQoL was moderately positively correlated with Activities of Daily Living (UPDRS II, PCC $=0.44)$, non-motor symptoms $(0.48)$, and measures of sleep $(0.50$ and 0.40$)$; all $p<0.001$. After 12 months of treatment with LCIG, improvements in HRQoL were moderately positively correlated with improvement from baseline in non-motor symptoms (PCC $=0.42)$, sleep $(0.54)$, and daytime sleepiness $(0.40$; all $p<0.001)$, and weakly correlated with improvement in dyskinesia signs and symptoms $(\mathrm{PCC}=0.23 ; p=0.011)$. Improvement in HRQoL was not correlated with improvements in OFF time or dyskinesia time.

Conclusion: Both at baseline and for change from baseline at 12 months, HRQoL was correlated with baseline and change from baseline in dyskinesia, Activities of Daily Living, and non-motor symptoms, including sleep; but not with baseline or change in OFF time.

Keywords: Dyskinesia, health-related quality of life, levodopa-carbidopa intestinal gel, non-motor symptoms, Parkinson's disease

\section{INTRODUCTION}

Levodopa is the gold standard for managing Parkinson's disease (PD), but it has a short plasma half-life that can result in motor fluctuations and dyskinesia [1-3]. One solution to address the short half-life of levodopa for patients with advanced PD has been the development of continuous levodopa infusion with levodopa-carbidopa intestinal gel (LCIG). LCIG has been shown in many studies to improve motor and non-motor fluctuations, dyskinesia, and health-related quality of life (HRQoL) in patients with advanced PD [4-7].

Findings from some studies have shown that motor symptoms and dyskinesia have a significant impact on HRQoL [8-10]. However, results in other studies have shown no correlation or only weak correlations [11, 12], still other studies have been limited by methodological and statistical limitations [13-19]. Non-motor symptoms have also been shown to impact HRQoL, suggesting that the effects of treatment on HRQoL may be more complex than improvements resulting from changes in motor symptoms alone [10, 14-16, 18].

DUOdopa/Duopa in Patients with Advanced Parkinson's Disease-a GLobal OBservational Study Evaluating Long-Term Effectiveness (DUOGLO BE) - is an ongoing long-term effectiveness study of the real-world use of LCIG. While the study is still in progress, results from the second interim analysis of the safety and efficacy of LCIG have been published [20]. These data are the basis of the planned analysis reported here. After 12 months, there was a significant $(3.9 \pm 3.6 \mathrm{~h} /$ day; $p \leq 0.001)$ decrease in OFF time, as well as improvements in the signs and symptoms of dyskinesia (Unified Dyskinesia Rating Scale [UDysRS] total score; $p \leq 0.001$ ). Non-motor symptoms (Non-Motor Symptoms Scale
[NMSS]), measures of sleep (Parkinson's Disease Sleep Scale [PDSS-2] and NMSS sleep subdomain; both $p \leq 0.001$ ), and daytime somnolence (Epworth Sleepiness Scale $[\mathrm{ESS}] p=0.042$ ) all showed significant improvements [20]. However, changes in Activities of Daily Living (Unified Parkinson's Disease Rating Scale [UPDRS II]) and motor symptoms (UPDRS III) were not significant. Importantly, scores on the Parkinson's Disease Questionnaire-8 (PDQ8) summary index, which measures HRQoL, were improved by -9.0 points $(p<0.001)$, a substantially greater change than the minimum clinically important difference of -5.94 [21]. This report from the second interim analysis of DUOGLOBE examines the correlation of HRQoL with symptoms of PD at baseline and after 12 months treatment with LCIG.

\section{METHODS}

\section{Study design and treatment}

DUOGLOBE is an ongoing 3-year, international, prospective, single-arm, post-marketing observational study designed to assess the longterm effectiveness of LCIG in patients with advanced PD (https://www.clinicaltrials.gov identifier: NCT02611713). This study is being conducted at 55 movement disorders centers in Australia, Belgium, Hungary, Israel, Italy, Romania, Slovenia, Spain, United Kingdom, and the United States. The study was approved by an independent ethics committee/institutional review board at each site. Here we describe a planned analysis based on data from the second interim analysis. The overall design of DUOGLOBE has been published in detail previously and is described briefly below [20]. 


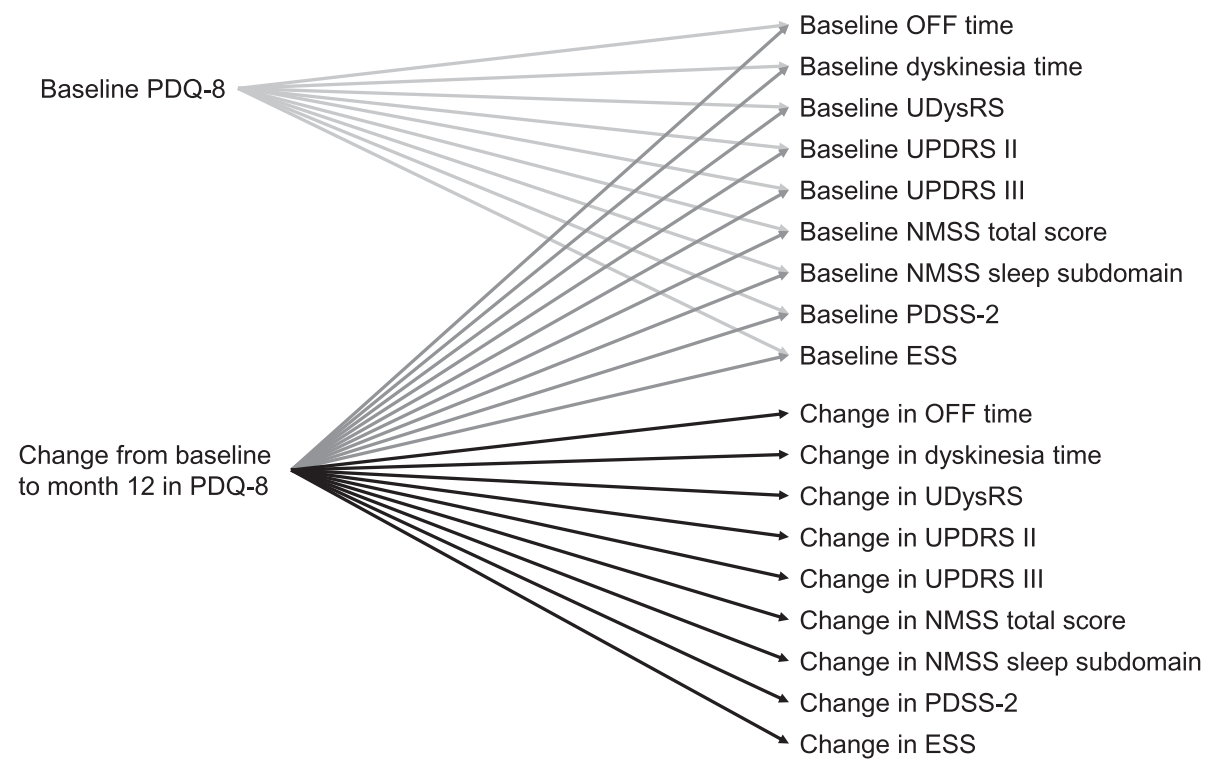

Fig. 1. Correlations analyzed. ESS, Epworth Sleepiness Scale; NMSS, Non-Motor Symptom Scale; PDQ-8, 8-item Parkinson's Disease Questionnaire; PDSS-2, Parkinson's Disease Sleep Scale-2; UDysRS, Unified Dyskinesia Rating Scale; UPDRS, Unified Parkinson's Disease Rating Scale.

\section{Patients}

Patients enrolled in the study must have been eligible for LCIG therapy in accordance with the approved local label and reimbursement criteria in the participating country. Exclusion criteria included previous surgery for PD, including deep brain stimulation (permitted in the United States only after a protocol amendment); current treatment with continuous apomorphine infusion; and/or a Mini-Mental State Examination score $<24$.

\section{Assessments}

The primary efficacy measure for DUOGLOBE was the change in the number of hours spent in OFF time as reported by the patient for the day prior to the study visit. Other assessments in this analysis included baseline and change from baseline at 12 months in the duration of dyskinesia as reported by the patient; UPDRS part II and part III scores; UDysRS total score and subdomain scores; NMSS total score and subdomain scores, including the sleep subdomain; PDSS-2 total score that measures sleep quality; ESS total score that measures daytime somnolence; and PDQ-8 summary index score. The purpose of this analysis was to assess the correlations between HRQoL (PDQ-8 summary index), and the measures listed above.

\section{Statistics}

Demographics and baseline characteristics are presented using descriptive statistics. For this planned analysis, Pearson correlation coefficients were calculated using non-missing data for baseline correlations of HRQoL (PDQ-8) and motor symptoms (OFF time, UDysRS total score, duration of dyskinesia, UPDRS part III), Activities of Daily Living (UPDRS part II), and non-motor symptoms (NMSS total score, NMSS sleep subdomain score, PDSS-2 total score, ESS total score), as well as for change from baseline to month 12 for HRQoL and the above-mentioned outcomes (Fig. 1). Statistical significance was calculated using a two-sided test. All statistical analyses were conducted using SAS version 9.4 (SAS Institute, Cary, NC, USA).

\section{RESULTS}

\section{Patients}

A total of 195 patients, with a mean (standard deviation) age of $70.2(8.2)$ years and a mean $11.2(4.8)$ years since diagnosis, were enrolled in the study. Baseline characteristics and patient demographics were reported previously [20], and are summarized in Table 1. 


\section{Correlation of HRQoL at baseline with baseline measures of PD severity}

At baseline, there was a moderate positive correlation between HRQoL, as measured by PDQ-8,

Table 1

Baseline demographics and characteristics

\begin{tabular}{lcc}
\hline Characteristic & $n$ & LCIG \\
\hline Age $^{*}, \mathrm{y}$ & 195 & $70.2 \pm 8.2$ \\
Male* $^{*}, \mathrm{n}(\%)$ & 195 & $120(61.5)$ \\
BMI $^{*}, \mathrm{~kg} / \mathrm{m}^{2}$ & 182 & $25.9 \pm 4.1$ \\
PD duration $^{*}, \mathrm{n}(\%)$ & & \\
$\quad<10 \mathrm{y}$ & 195 & $94(48.5)$ \\
$\quad \geq 10 \mathrm{y}$ & 195 & $100(51.5)$ \\
Time from diagnosis to LCIG initiation* $^{*}, \mathrm{y}$ & 194 & $11.2 \pm 4.8$ \\
OFF time $^{\dagger}, \mathrm{h}_{\text {/day }}$ & 164 & $6.0 \pm 3.4$ \\
Dyskinesia time $^{\dagger}, \mathrm{h}$ & 165 & $4.1 \pm 3.7$ \\
PDQ-8 summary index $^{\dagger}$ & 171 & $45.1 \pm 18.1$ \\
UDysS score $^{\dagger}$ & 152 & $33.7 \pm 21.1$ \\
UPDRS II $^{\dagger}$ & 173 & $14.8 \pm 7.8$ \\
UPDRS III $^{\dagger}$ & 171 & $27.6 \pm 13.2$ \\
NMSS $^{\dagger}$ & 162 & $87.9 \pm 51.3$ \\
NMSS Sleep subdomain $^{\dagger}$ & 169 & $16.3 \pm 10.5$ \\
PDSS-2 $^{\dagger}$ & 171 & $26.6 \pm 11.7$ \\
ESS $^{\dagger}$ & 172 & $9.8 \pm 5.3$ \\
\hline All $^{\dagger}$
\end{tabular}

All values are mean \pm standard deviation, unless otherwise specified. *Safety population. ${ }^{\dagger}$ Full analysis set. BMI, body mass index; ESS, Epworth Sleepiness Scale; LCIG, levodopa-carbidopa intestinal gel; NMSS, Non-Motor Symptom Scale; PD, Parkinson's disease; PDQ-8, 8-item Parkinson's Disease Questionnaire; PDSS-2, Parkinson's Disease Sleep Scale-2; UDysRS, Unified Dyskinesia Rating Scale; UPDRS, Unified Parkinson's Disease Rating Scale. and baseline Activities of Daily Living (UPDRS II; Pearson coefficient $=0.44$ ), non-motor symptoms (NMSS; Pearson coefficient $=0.48$ ), and sleep (PDSS-2 and NMSS sleep subdomain; Pearson coefficients $=0.50$ and 0.40 , respectively); all $p<0.001$ (Fig. 2). PDQ-8 scores were better (lower scores) with better UPDRS II, UDysRS, PDSS-2, and NMSS scores (lower scores; scatter plots shown in Fig. 3). In addition, there was a statistically significant, although weak, positive correlation between baseline UDysRS and baseline PDQ- 8 scores (Pearson correlation coefficient $=0.27 ; p<0.001$ ). Baseline HRQoL was not correlated with baseline OFF time or dyskinesia time.

\section{Correlation of baseline parameters with improvements in $H R Q o L$}

As reported previously [20], mean (standard deviation) scores on the PDQ- 8 questionnaire were significantly improved from baseline to month $12(-9.0 \pm 21.6 ; p<0.001)$. Correlations between baseline parameters and improvements in PDQ-8 questionnaire scores were statistically significant, though weak. The strongest relationships were negative correlations between absolute changes in PDQ-8 questionnaire scores and baseline PDSS-2 scores (correlation coefficient $-0.32 ; p<0.001$ ), NMSS total score, NMSS sleep subdomain scores (both -0.3 ; $p<0.001)$, and ESS scores $(-0.22 ; p=0.01)$, meaning that worse baseline scores correlated with greater improvements in PDQ-8 after initiation of LCIG, making those baseline parameters weak predictors

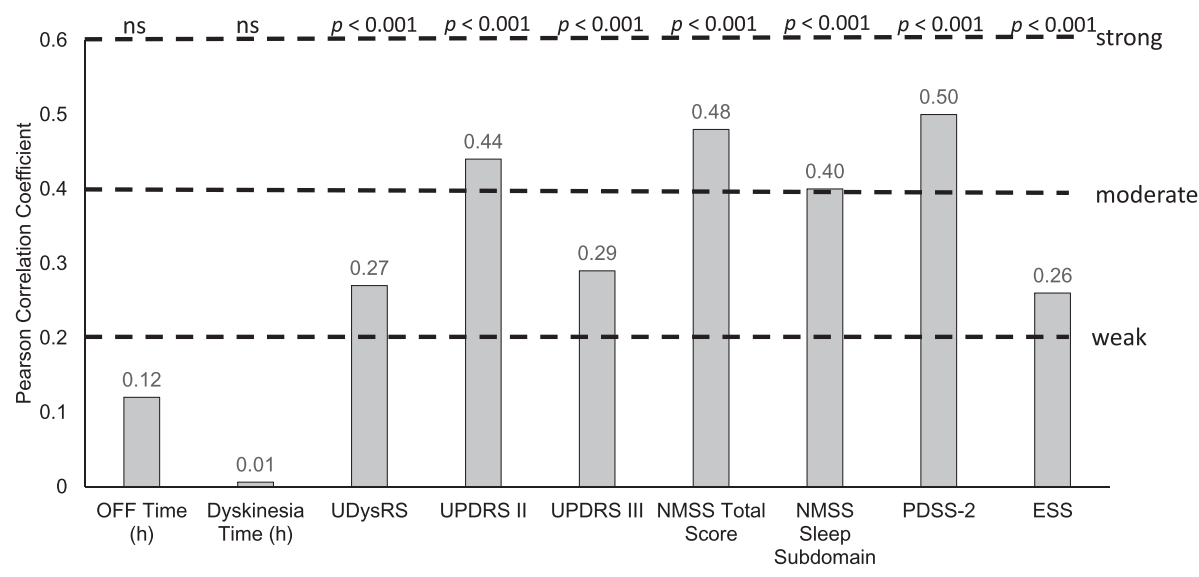

Fig. 2. Correlation of baseline parameters with PDQ-8 at baseline. ESS, Epworth Sleepiness Scale; h, hour; NMSS, Non-Motor Symptom Scale; ns, not significant; PDQ-8, 8-item Parkinson's Disease Questionnaire; PDSS-2, Parkinson's Disease Sleep Scale-2; UDysRS, Unified Dyskinesia Rating Scale; UPDRS, Unified Parkinson's Disease Rating Scale. 

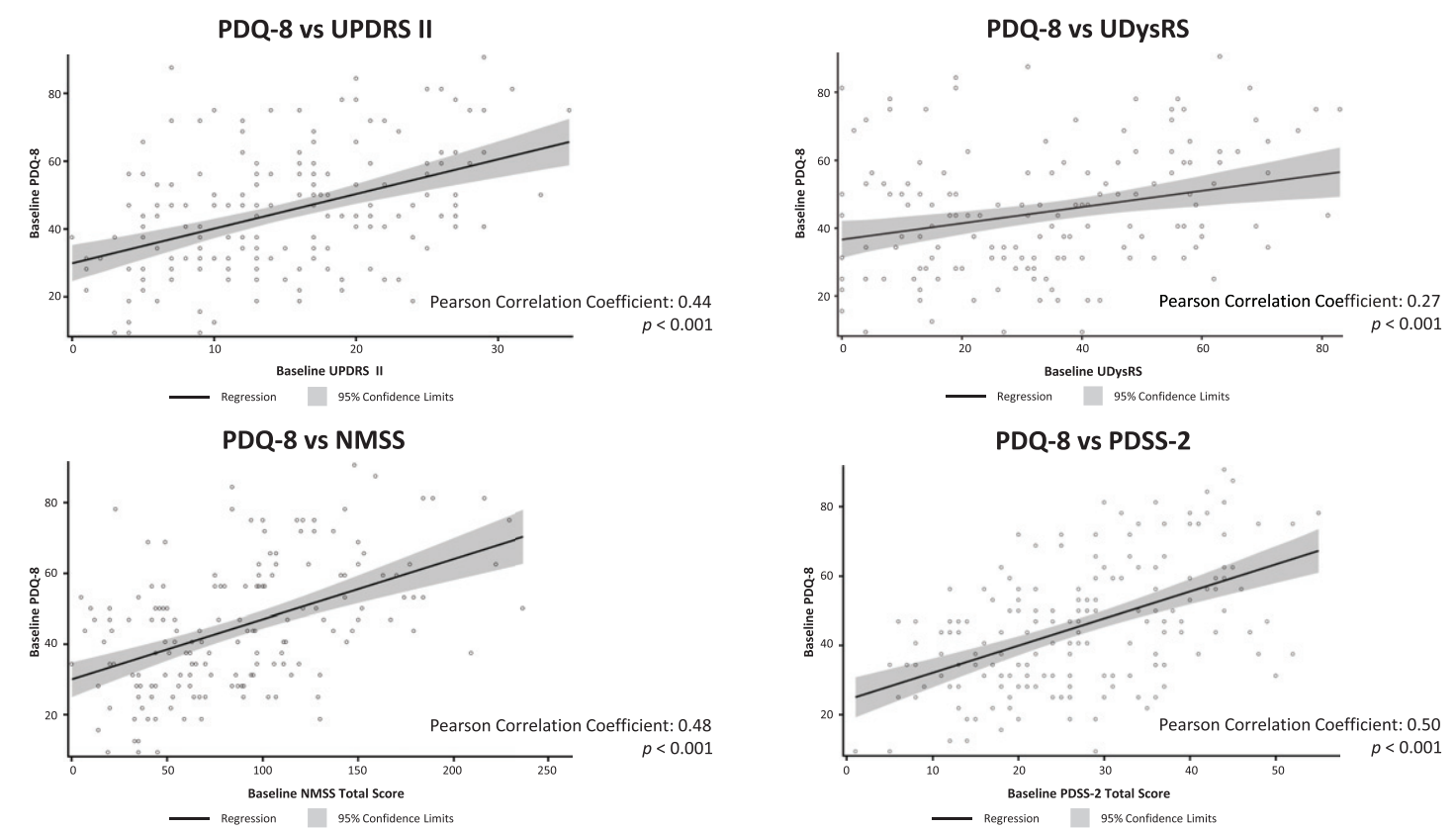

Fig. 3. Scatter plots of correlation of PDQ-8 at baseline with baseline UPDRS II, UDysRS, NMSS, and PDSS-2. NMSS, Non-Motor Symptom Scale; ns, not statistically significant; PDQ-8, 8-item Parkinson's Disease Questionnaire; PDSS-2, Parkinson's Disease Sleep Scale-2; UDysRS, Unified Dyskinesia Rating Scale; UPDRS, Unified Parkinson's Disease Rating Scale.

of HRQoL improvement. All other correlations were not statistically significant.

\section{Correlation of changes in HRQoL with changes in disease parameters after $L C I G$}

After 12-months of treatment with LCIG, absolute changes in PDQ-8 summary index scores were moderately positively correlated with change from baseline in non-motor symptoms (NMSS: Pearson correlation coefficient $=0.42 ; p<0.001$ ), sleep (PDSS-2, 0.54; $p<0.001$ ), and daytime sleepiness scores (ESS, 0.40; $p<0.001$; Figs. 4 and 5). There were statistically significant weak positive correlations between changes in PDQ-8 summary index scores and changes in UPDRS II scores $(0.33$; $p<0.001)$, UDysRS scores $(0.23 ; p=0.011)$, and the NMSS sleep subdomain $(0.37 ; p<0.001 ;$ Figs. 4 and 5). Changes in PDQ-8 summary index scores were not correlated with improvements in OFF time or dyskinesia time.

\section{Safety}

The safety results from this study have been previously reported [20]. Serious adverse events are summarized in Table 2. The most frequent serious
Table 2

Overview of safety events

\begin{tabular}{lc}
\hline Overview & Total \\
& $(N=195)$ \\
\hline SAEs & $79(40.5)$ \\
SAEs possibly related to LCIG & $23(11.8)$ \\
AEs leading to drug being withdrawn & $29(14.9)$ \\
Severe AEs & $46(23.6)$ \\
Deaths & $13(6.7)$ \\
$\quad$ Deaths possibly related to LCIG treatment* & $1(0.5)^{\dagger}$ \\
\hline MedDRA preferred term & Treatment- \\
& emergent \\
& SAEs ${ }^{\ddagger}$ \\
\hline Fall & $6(3.1)$ \\
Urinary tract infection & $6(3.1)$ \\
Hip fracture & $5(2.6)$ \\
Pneumonia & $5(2.6)$ \\
Abdominal pain & $4(2.1)$ \\
\hline
\end{tabular}

${ }^{*}$ As assessed by the study investigator. ${ }^{\dagger}$ Abdominal obstruction. ${ }^{\ddagger}$ Occurring in $\geq 2 \%$ of patients by MedDRA preferred term. Data are presented as $n(\%)$. AE, adverse event; LCIG, levodopacarbidopa intestinal gel; MedDRA, Medical Dictionary for Regulatory Activities, version 21.1; SAE, serious adverse event.

adverse events were fall $(3.1 \%[n=6])$ and urinary tract infection $(3.1 \%[n=6])$. There were 13 deaths, of which one death (abdominal obstruction) was believed by the investigator to be related to the study treatment. 


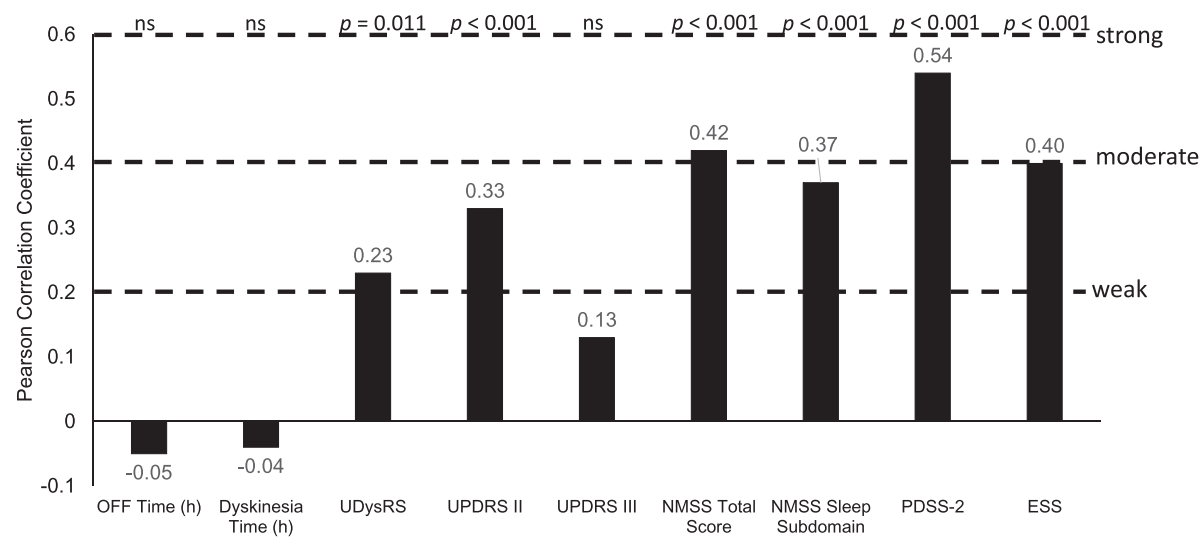

Fig. 4. Correlation of change from baseline in efficacy parameters with PDQ-8 change from baseline. ESS, Epworth Sleepiness Scale; h, hour; NMSS, Non-Motor Symptom Scale; ns, not significant; PDQ-8, 8-item Parkinson's Disease Questionnaire; PDSS-2, Parkinson's Disease Sleep Scale-2; UDysRS, Unified Dyskinesia Rating Scale; UPDRS, Unified Parkinson's Disease Rating Scale.
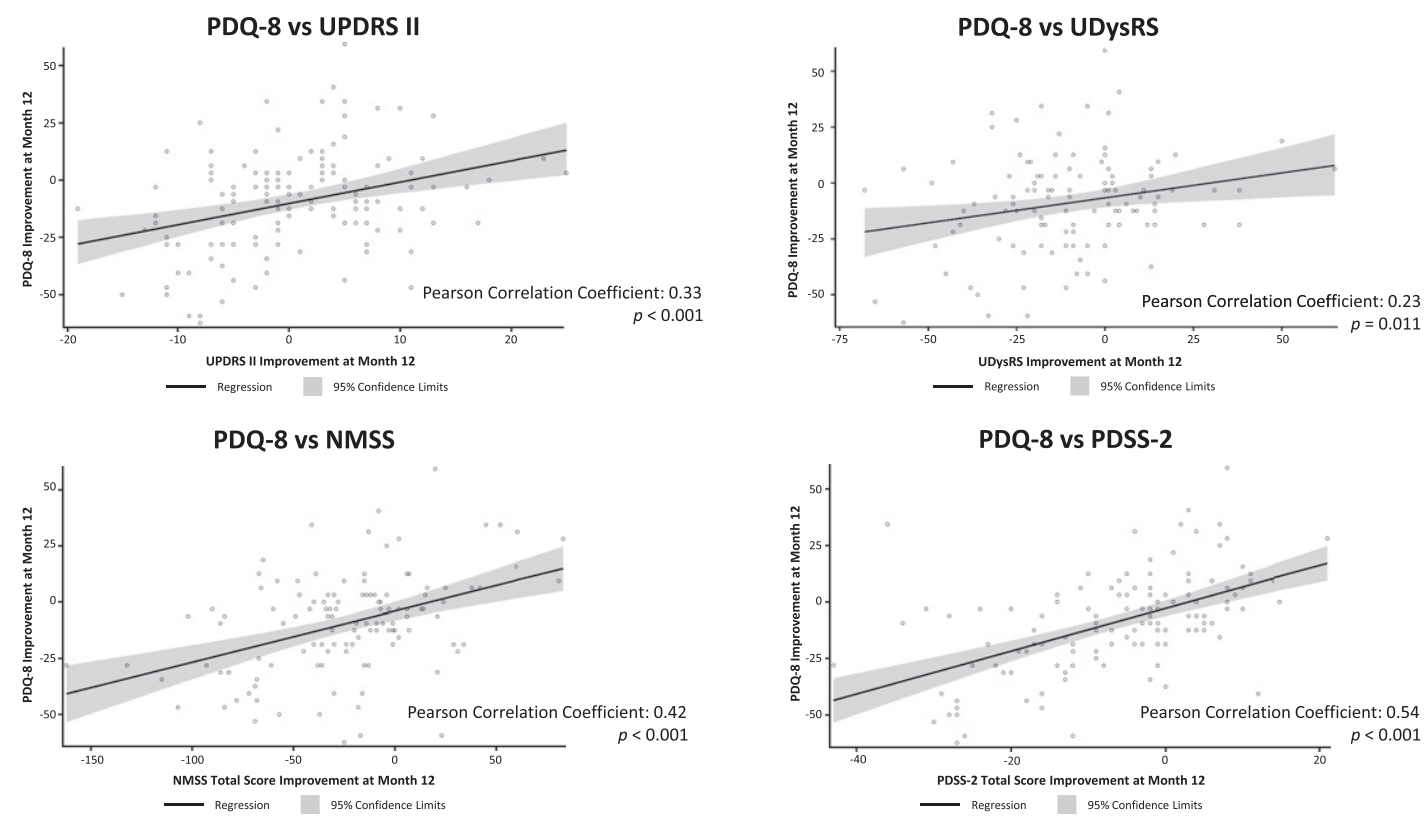

Fig. 5. Scatter plots of correlation of change from baseline in PDQ-8 with change from baseline in UPDRS II, UDysRS, NMSS, and PDSS-2. NMSS, Non-Motor Symptom Scale; ns, not statistically significant; PDQ-8, 8-item Parkinson's Disease Questionnaire; PDSS-2, Parkinson's Disease Sleep Scale-2; UDysRS, Unified Dyskinesia Rating Scale; UPDRS, Unified Parkinson's Disease Rating Scale.

\section{DISCUSSION}

DUOGLOBE is the first large-scale, prospective, long-term study to simultaneously assess how dyskinesia, motor symptoms, and non-motor symptoms impact HRQoL in patients with advanced PD. Our data show that non-motor symptoms are positively correlated with HRQoL both before treatment and following the start of treatment. There was a moderate positive correlation between measures of non-motor symptoms, including sleep and daytime sleepiness at baseline and PDQ- 8 summary index scores at baseline. This is consistent with results from previous studies that have shown that worsening non-motor symptoms, including sleep, significantly worsen HRQoL to a greater extent than do motor symptoms [10, 14-16, 18, 22]. It is evident, therefore, that HRQoL is affected by many factors beyond 
dyskinesia and motor symptoms alone in patients with advanced PD. Importantly, treatment with LCIG improved both non-motor symptoms and HRQoL [5-7]. In our analysis, improvements in HRQoL in patients treated with LCIG are most strongly correlated with improvements in non-motor symptoms, especially sleep. Further evidence to support this finding comes from the GLORIA registry that indicates that the burden of non-motor symptoms at baseline predicts improvement in HRQoL in patients treated with LCIG [23].

Results from this analysis also demonstrate that there is a moderate positive correlation between Activities of Daily Living (UPDRS II scores) at baseline and HRQoL (PDQ-8 summary index scores) at baseline, which is in line with data presented in previous studies [9-11]. Changes in UPDRS II scores showed a weak positive correlation with changes in HRQoL. Using the short PDQ-8 tool to assess patients with advanced PD may give clinicians a good overview of the effect of PD on HRQoL and thereby indirectly also on Activities of Daily Living. The PDQ-8 questionnaire may also help to identify those patients whose PD is not well controlled with their current medication.

There was a statistically significant, but only weak, positive correlation between the signs and symptoms of dyskinesia (UDysRS total score) at baseline and HRQoL at baseline. Because UDysRS has been introduced more recently and is now increasingly used to assess the anti-dyskinetic efficacy of treatments, this weak correlation was a novel finding [24]. However, although the correlation was weak, treatment with LCIG for 1 year in this interim dataset was associated with significant improvements in the signs and symptoms of dyskinesia (UDysRS total score) [20]; these findings are also consistent with results from an open-label Hungarian registry [25].

In many studies, treatment with LCIG has been shown to improve OFF time in a sustained and highly statistically significant manner, just as it did in this study [4-7]. Surprisingly, there was no correlation between changes in PDQ-8 summary index scores and improvements in OFF time or dyskinesia time while patients were receiving LCIG treatment. Studies of some other treatments for PD have indicated at least weak correlations between dyskinesia, and ON-OFF fluctuations, and HRQoL [8, 11-19]. For example, following deep brain stimulation of the subthalamic nucleus, improvements in HRQoL were influenced by cumulative OFF time, UPDRS III ON/OFF, and UPDRS dyskinesia score in ON time [8]. Our observations with the use of LCIG in this study lead us to conclude that although continuous levodopa treatment usually produces substantial improvements in motor symptoms, the benefits in terms of HRQoL are not derived from these motor effects alone, but rather depend in large part on the effects of LCIG on non-motor symptoms, sleep, and daytime sleepiness.

The present data are insufficient to offer specific recommendations about selecting patients for treatment, but they do suggest that in the population of patients with advanced PD enrolled by the investigators in this study, it is reasonable to expect LCIG to lead to improvements in both motor and non-motor symptoms, and to have an overall beneficial effect on HRQoL.

A strength of this analysis is that data are collected from a large, prospective study in patients with advanced PD who have undergone long-term follow-up and comprehensive assessments of motor complications and non-motor symptoms, although some of these measures could be subject to recall bias. These long-term follow-up data make it possible to evaluate correlations between HRQoL and other motor and non-motor symptoms both at baseline and during treatment with LCIG. However, there are also limitations. Despite the robustness of the DUOGLOBE study, the study is not large enough to conduct meaningful multi-variable analyses to further refine assessments of how these motor and non-motor symptoms might jointly affect HRQoL. Another limitation is that this was an open-label, noncontrolled, single-arm study. These results also do not provide insight into the impact that adverse events may have on HRQoL. Given the non-randomized nature of the study and the high probability of potential confounders missing in the data, we abstained from conducting mediation analyses where much stronger assumptions including sequential ignorability or exchangeability, or no unmeasured confounders are required in the analysis but are unlikely to be achieved in this non-controlled, non-randomized observational study. A multi-variable regression analysis was not pursued due to the co-linearity issues from highly correlated motor and non-motor symptoms in the study.

Findings from this analysis have demonstrated that after LCIG treatment in advanced PD, changes in HRQoL are positively correlated with changes in Activities of Daily Living as measured by UPDRS II scores, non-motor symptoms, and sleep. These observations point to the importance of considering 
effects on non-motor features when evaluating the effectiveness of treatments for PD. The safety findings reported in the 1-year interim analysis of the DUOGLOBE study [20] were consistent with the established safety profile of LCIG.

\section{ACKNOWLEDGMENTS}

AbbVie Inc. participated in the study design; study research; collection, analysis, and interpretation of data; and writing, reviewing, and approving this manuscript for submission. AbbVie funded the research for this study and provided writing support for this manuscript. No honoraria or payments were made for authorship. Medical writing assistance, funded by AbbVie, was provided by James Street, PhD, Alicia Salinero, PhD, and Kersten Reich, MPH, CMPP, of JB Ashtin.

\section{CONFLICT OF INTEREST}

Dr Kovács was a study investigator and has received honorarium from AbbVie, Abbott, Boston Scientific, Boehringer Ingelheim, GSK Pharmaceuticals, KRKA, Medtronic, Teva, and UCB Pharma, and has also received honoraria for lecturing at symposia. He has been a consultant for AbbVie, Abbott, KRKA, and Teva. He has received research funding from the Hungarian National Research, Development and Innovation Office; University of Pécs; and Medtronic. Dr Anca-Herschkovitsch is a study investigator and consultant for AbbVie.

Dr Cubo is a study investigator and has received honoraria for consulting from AbbVie, and travel grants from Allergan and Boston Scientific. Dr Davis is a study investigator and has received grant/research support from the Parkinson Foundation and the Peterson Foundation for Parkinsons, and honoraria for consulting from AbbVie. Dr Iansek is a study investigator and has received honorarium for consulting from AbbVie. Dr Siddiqui is a study investigator. $\mathrm{He}$ has received research support as a principal investigator from Sunovion Pharma, AbbVie, Boston Scientific Inc, Biogen MA, Theravance Biopharma, Michael J Fox Foundation / Neuropoint Alliance, Impax Laboratories, Neuraly, and Sun Pharma. He has served as a consultant and/or scientific advisor for Boston Scientific and AbbVie. Dr Simu is a study investigator and has received honoraria for lectures at symposia and consultant fees from Teva, AbbVie, Merck, Servier Pharma, AOP Orphan, Boehringer
Ingelheim, Sanofi, KRKA, Roche, Novartis, and UCB Pharma. Dr Standaert is a member of the faculty of the University of Alabama at Birmingham and is supported by endowment and University funds. $\mathrm{He}$ is an investigator in studies funded by AbbVie; the American Parkinson Disease Association; the Michael J. Fox Foundation for Parkinson Research; Alabama Department of Commerce; the Department of Defense; and NIH grants P50NS108675, R25NS079188, and T32NS095775. He has a clinical practice and is compensated for these activities through the University of Alabama Health Services Foundation. In addition, since 1 January 2020 he has served as a consultant for or received honoraria from AbbVie, Curium, Sutter Health, the Warren Alpert Foundation, the International Parkinson Disease and Movement Disorder Society, Theravance, McGraw Hill, and Sanofi-Aventis.

Dr Chaudhuri is a study investigator and has served as an advisory board member for AbbVie, UCB, GKC, Bial, Cynapsus, Lobsor, Stada, Medtronic, Zambon, Profile, Sunovion, Roche, Therevance, Scion, Britannia, Acadia, and 4D. He received honoraria for lectures from AbbVie, Britannia, UCB, Zambon, Novartis, Boeringer Ingelheim, Bial, Kyowa Kirin, and SK Pharma. He has received grants (investigator initiated) from Britannia Pharmaceuticals, AbbVie, UCB, GKC, and Bial; and academic grants from EU, IMI EU, Horizon 2020, Parkinson's UK, NIHR, PDNMG, EU (Horizon 2020), Kirby Laing Foundation, NPF, MRC, and Wellcome Trust. He receives royalties from Oxford University Press and holds intellectual property rights for the King's Parkinson's Pain Scale and Parkinson's Disease Sleep Scale. Dr Bourgeois has received honoraria for consulting from AbbVie. Dr Pontieri is a member of the faculty at the Sapienza University of Rome and has a clinical practice at Sant'Andrea Hospital in Rome. He is also Senior Research Neurologist at the Fondazione Santa Lucia, IRCCS, Rome. He is an investigator in studies funded by AbbVie, Zambon, and the Italian Ministry of Health and has served as consultant for or received honoraria from AbbVie, Zambon, FB Health, Bial, and Lundbeck. Dr Aldred has received honoraria from Abbott Laboratories, AbbVie, Accorda, Allergan, Medtronic, Boston Scientific, Teva, and US World Meds. He has received research funding for studies sponsored by AbbVie, Acadia, Annexon, Amneal/Impax, Biogen, Boston Scientific, Centogene, Denali, Massachusetts General Hospital, Neuroderm, Neurocrine, Northwestern University, Sunovion, Triplet Therapeutics, 
and Theravance. He is a scientific advisor for AbbVie, Allergan, and Boston Scientific. Dr Bergmann, Dr Gao, and Dr Kukreja are employees of AbbVie and may hold AbbVie stock and/or stock options.

\section{REFERENCES}

[1] Rao SS, Hofmann LA, Shakil A (2006) Parkinson's disease: Diagnosis and treatment. Am Fam Physician 74, 2046-2054.

[2] Miyasaki JM, Martin W, Suchowersky O, Weiner WJ, Lang AE (2002) Practice parameter: Initiation of treatment for Parkinson's disease: An evidence-based review: Report of the Quality Standards Subcommittee of the American Academy of Neurology. Neurology 58, 11-17.

[3] Fox SH, Katzenschlager R, Lim SY, Barton B, de Bie RMA, Seppi K, Coelho M, Sampaio C, Movement Disorder Society Evidence-Based Medicine Committee (2018) International Parkinson and Movement Disorder Society evidence-based medicine review: Update on treatments for the motor symptoms of Parkinson's disease. Mov Disord 33, 1248-1266.

[4] Antonini A, Fung VS, Boyd JT, Slevin JT, Hall C, Chatamra K, Eaton S, Benesh JA (2016) Effect of levodopa-carbidopa intestinal gel on dyskinesia in advanced Parkinson's disease patients. Mov Disord 31, 530-537.

[5] Antonini A, Poewe W, Chaudhuri KR, Jech R, Pickut B, Pirtošek Z, Szasz J, Valldeoriola F, Winkler C, Bergmann L, Yegin A, Onuk K, Barch D, Odin P (2017) Levodopacarbidopa intestinal gel in advanced Parkinson's: Final results of the GLORIA registry. Parkinsonism Relat Disord 45, 13-20.

[6] Fernandez HH, Standaert DG, Hauser RA, Lang AE, Fung VS, Klostermann F, Lew MF, Odin P, Steiger M, Yakupov EZ, Chouinard S, Suchowersky O, Dubow J, Hall CM, Chatamra K, Robieson WZ, Benesh JA, Espay AJ (2015) Levodopa-carbidopa intestinal gel in advanced Parkinson's disease: Final 12-month, open-label results. Mov Disord 30, 500-509.

[7] Standaert DG, Rodriguez RL, Slevin JT, Lobatz M, Eaton S, Chatamra K, Facheris MF, Hall C, Sail K, Jalundhwala YJ, Benesh J (2017) Effect of levodopa-carbidopa intestinal gel on non-motor symptoms in patients with advanced Parkinson's disease. Mov Disord Clin Pract 4, 829-837.

[8] Daniels C, Krack P, Volkmann J, Raethjen J, Pinsker MO, Kloss M, Tronnier V, Schnitzler A, Wojtecki L, Botzel K, Danek A, Hilker R, Sturm V, Kupsch A, Karner E, Deuschl G, Witt K (2011) Is improvement in the quality of life after subthalamic nucleus stimulation in Parkinson's disease predictable? Mov Disord 26, 2516-2521.

[9] Ellis T, Cavanaugh JT, Earhart GM, Ford MP, Foreman KB, Dibble LE (2011) Which measures of physical function and motor impairment best predict quality of life in Parkinson's disease? Parkinsonism Relat Disord 17, 693-697.

[10] Skorvanek M, Martinez-Martin P, Kovacs N, Zezula I, Rodriguez-Violante M, Corvol JC, Taba P, Seppi K, Levin O, Schrag A, Aviles-Olmos I, Alvarez-Sanchez M, Arakaki T, Aschermann Z, Benchetrit E, Benoit C, Bergareche-Yarza A, Cervantes-Arriaga A, Chade A, Cormier F, Datieva V, Gallagher DA, Garretto N, Gdovinova Z, Gershanik O, Grofik M, Han V, Kadastik-Eerme L, Kurtis MM, Mangone G, Martinez-Castrillo JC, Mendoza-Rodriguez A, Minar M, Moore HP, Muldmaa M, Mueller C, Pinter B, Poewe W, Rallmann K, Reiter E, Rodriguez-Blazquez C, Singer C,
Valkovic P, Goetz CG, Stebbins GT (2018) Relationship between the MDS-UPDRS and quality of life: A large multicenter study of 3206 patients. Parkinsonism Relat Disord 52, 83-89.

[11] Chapuis S, Ouchchane L, Metz O, Gerbaud L, Durif F (2005) Impact of the motor complications of Parkinson's disease on the quality of life. Mov Disord 20, 224-230.

[12] Schrag A, Jahanshahi M, Quinn N (2000) What contributes to quality of life in patients with Parkinson's disease? $J$ Neurol Neurosurg Psychiatry 69, 308-312.

[13] Wu J, Lim EC, Nadkarni NV, Tan EK, Kumar PM (2019) The impact of levodopa therapy-induced complications on quality of life in Parkinson's disease patients in Singapore. Sci Rep 9, 9248.

[14] Rahman S, Griffin HJ, Quinn NP, Jahanshahi M (2008) Quality of life in Parkinson's disease: The relative importance of the symptoms. Mov Disord 23, 1428-1434.

[15] Winter Y, von Campenhausen S, Gasser J, Seppi K, Reese JP, Pfeiffer KP, Botzel K, Oertel WH, Dodel R, Poewe W (2010) Social and clinical determinants of quality of life in Parkinson's disease in Austria: A cohort study. J Neurol 257, 638-645.

[16] Winter $\mathrm{Y}$, von Campenhausen S, Arend M, Longo K, Boetzel K, Eggert K, Oertel WH, Dodel R, Barone P (2011) Health-related quality of life and its determinants in Parkinson's disease: Results of an Italian cohort study. Parkinsonism Relat Disord 17, 265-269.

[17] Hechtner MC, Vogt T, Zollner Y, Schroder S, Sauer JB, Binder H, Singer S, Mikolajczyk R (2014) Quality of life in Parkinson's disease patients with motor fluctuations and dyskinesias in five European countries. Parkinsonism Relat Disord 20, 969-974.

[18] Kurihara K, Nakagawa R, Ishido M, Yoshinaga Y, Watanabe J, Hayashi Y, Mishima T, Fujioka S, Tsuboi Y (2020) Impact of motor and nonmotor symptoms in Parkinson disease for the quality of life: The Japanese Quality-of-Life Survey of Parkinson Disease (JAQPAD) study. J Neurol Sci 419, 117172.

[19] Gulunay A, Cakmakli GY, Yon MI, Ulusoy EK, Karakoc M (2020) Frequency of non-motor symptoms and their impact on the quality of life in patients with Parkinson's disease: A prospective descriptive case series. Psychogeriatrics 20, 206-211.

[20] Standaert DG, Aldred J, Anca-Herschkovitsch M, Bourgeois P, Cubo E, Davis TL, Iansek R, Kovács N, Pontieri FE, Siddiqui M, Simu M, Bergmann L, Kukreja P, Robieson WZ, Chaudhuri KR (2021) DUOGLOBE: One year outcomes in a real world study of levodopa-carbidopa intestinal gel for Parkinson's disease. Mov Disord Clin Pract 8, 10611074.

[21] Horvath K, Aschermann Z, Kovacs M, Makkos A, Harmat M, Janszky J, Komoly S, Karadi K, Kovacs N (2017) Changes in quality of life in Parkinson's disease: How large must they be to be relevant? Neuroepidemiology 48, 1-8.

[22] Berganzo K, Tijero B, Gonzalez-Eizaguirre A, Somme J, Lezcano E, Gabilondo I, Fernandez M, Zarranz JJ, GomezEsteban JC (2016) Motor and non-motor symptoms of Parkinson's disease and their impact on quality of life and on different clinical subgroups. Neurologia 31, 585-591.

[23] Chaudhuri KR, Antonini A, Robieson WZ, SanchezSolino O, Bergmann L, Poewe W (2019) Burden of non-motor symptoms in Parkinson's disease patients predicts improvement in quality of life during treatment with levodopa-carbidopa intestinal gel. Eur J Neurol 26, 581e543. 
[24] Goetz CG, Stebbins GT, Chung KA, Hauser RA, Miyasaki JM, Nicholas AP, Poewe W, Seppi K, Rascol O, Stacy MA, Nutt JG, Tanner CM, Urkowitz A, Jaglin JA, Ge S (2013) Which dyskinesia scale best detects treatment response? Mov Disord 28, 341-346.

[25] Juhász A, Aschermann Z, Ács P, Janszky J, Kovács M, Makkos A, Harmat M, Tényi D, Karádi K, Komoly S, Takáts
A, Tóth A, Nagy H, Klivényi P, Dibó G, Dézsi L, Zádori D, Annus Á, Vécsei L, Varannai L, Kovács N (2017) Levodopa/carbidopa intestinal gel can improve both motor and non-motor experiences of daily living in Parkinson's disease: An open-label study. Parkinsonism Relat Disord 37, 79-86. 